\title{
Right subclavian steal syndrome in a patient with aberrant right subclavian artery, common carotid trunk and hypoplastic left vertebral artery
}

\author{
Boochi Babu Mannuva, Rajasekhar Durgaprasad, Vanajakshamma \\ Velam, Shashanka Chunduri, Vijayalajshmi Devi B
}

\begin{abstract}
Introduction: Subclavian steal syndrome refers to the reversal of vertebral blood flow in the setting of proximal subclavian or innominate artery severe stenosis or occlusion resulting in the symptoms of vertebrobasilar insufficiency related to exercise of the upper extremities. It is more common on the left side. Case report: We reported a case of subclavian steal syndrome on the right side in a patient with aberrant right subclavian artery (ARSCA), hypoplastic left vertebral artery and common carotid trunk (CCT) branching in to the left and right common carotid arteries. Conclusion: Till now, combination of ARSCA, CCT and left vertebral artery hypoplasia in the same person has not been reported and this combination causes ischemia in large area of brain if the patient develops hemodynamically significant stenosis or occlusion in both CCT and ARSCA proximal to the origin of right vertebral artery.
\end{abstract}

Boochi Babu Mannuva1, Rajasekhar Durgaprasad², Vanajakshamma Velam ${ }^{3}$, Shashanka Chunduri4, Vijayalajshmi Devi $B^{5}$

Affiliations: ${ }^{1} \mathrm{MD}$, Senior Resident, Department of Cardiology, SVIMS, Tirupati, A.P, India; ${ }^{2}$ MD, FACC, Dean, Professor \& Head, Department of Cardiology, SVIMS, Tirupati, A.P, India; ${ }^{3} \mathrm{MD}$, Professor, Department of Cardiology, SVIMS, Tirupati, A.P, India; ${ }^{4} \mathrm{MD}$, Assistant Professor of Cardiology, SVIMS, Tirupati, A.P, India; ${ }^{5} \mathrm{DMRD}, \mathrm{DNB}$, Associate professor of radiology, SVIMS, Tirupati, A.P, India.

Corresponding Author: Dr. Boochi Babu Mannuva, (Senior Resident), Dept. of Cardiology, Sri Venkateswara Institute of Medical Sciences, Tirupati -517507, Andhra Pradesh, India; Ph: +91-7382782540; Email: e.mbb99@gmail.com

Received: 02 March 2013

Accepted: 18 April 2013

Published: 01 August 2013
Keywords: Right subclavian steal syndrome, Aberrant right subclavian artery, Common carotid trunk, Hypoplastic left vertebral artery

$* * * * * * * * *$

Mannuva BB, Durgaprasad R, Velam V, Chunduri S, Devi VB. Right subclavian steal syndrome in a patient with aberrant right subclavian artery, common carotid trunk and hypoplastic left vertebral artery. International Journal of Case Reports and Images 2013;4(8):406-410.

$$
* * * * * * * * *
$$

doi:10.5348/ijcri-2013-08-343-CR-2

\section{INTRODUCTION}

Subclavian steal phenomenon (SSP) is associated with flow reversal in the vertebral artery ipsilateral to a hemodynamically significant stenosis or occlusion of the subclavian artery. Subclavian steal syndrome (SSS) implies the presence of significant symptoms due to arterial insufficiency in the brain (i.e., vertebrobasilar insufficiency) or upper extremity, which are supplied by the subclavian artery. Contorni in 1960, first described retrograde flow in the vertebral artery [1]. In 1961, Reivich first recognized the association between this phenomenon and neurologic symptoms [2]. Fisher coined the term 'subclavian steal syndrome' for this combination of retrograde vertebral flow and neurologic symptoms [3].

Most patients with subclavian artery stenosis are asymptomatic because of the abundant collateral blood supply in the head, neck and shoulder. Symptoms, when they occur, are due mainly to ischemia of the ipsilateral upper extremity. Exercise-induced arm pain, fatigue, coolness, paresthesias or numbness occurs in approximately one-third of patients, but ischemic and trophic changes are rare. Symptoms of vertebrobasilar ischemia (VBI) of brainstem (dizziness, vertigo, ataxia, 
disequilibrium, drop attacks, diplopia, nystagmus, graying of vision, hemianopia, bilateral visual blurring, and syncope) are uncommon and usually occur in patients with concurrent significant stenotic/occlusive disease involving cerebral circulation [4]. In some cases, reduced arterial flow resistance, during upper extremity exercise, can precipitate vertebrobasilar symptoms. Collateral blood supply and the capacity to increase collateral flow determine patients who develop neurologic symptoms.

\section{CASE REPORT}

A 53-year-old male with hypertension presented with right arm discomfort, dizziness and presyncope that occurred even with minimal exertion of right upper extremity for the last two months. There was no past history of coronary or vascular disease. Physical examination revealed blood pressures $130 / 70 \mathrm{mmHg}$ in the left arm and 100/6o mmHg in the right arm, with weak brachial, radial and ulnar pulses on the right side. No carotid bruit was auscultated. His 12-lead electrocardiogram was normal. Two dimensional echocardiography and color Doppler sonography showed that three great vessels were originated from aortic arch and their course could not be delineated because of poor window. Color Doppler sonography of vertebral arteries revealed hypoplasia of left one and normal right vertebral artery with retrograde flow on the right side (subclavian steal) and of subclavian arteries showed that severe stenosis in right subclavian artery just proximal to the origin of vertebral artery and dampened flow with prolonged acceleration time was noted in distal right subclavian, axillary, brachial, radial and ulnar arteries. Computed tomography angiography (CTA) of the head and neck revealed that there was a critical stenosis in aberrant right subclavian artery (ARSCA) proximal to the origin of normal right vertebral artery, left and right common carotid arteries arising from the common carotid trunk (CCT), normal left subclavian artery and hypoplastic left vertebral artery (Figure 1 and Figure 2). The patient subsequently underwent coronary and selective ARSCA angiography after informed consent was obtained. His coronary angiogram revealed normal epicardial coronary arteries and selective ARSCA angiogram showed severe stenosis in its proximal part (Figure 3). Later patient underwent successful right subclavian artery angioplasty with stenting.

\section{DISCUSSION}

Subclavian steal syndrome defined as the presence of significant symptoms due to ischemia in the brain (i.e., vertebrobasilar insufficiency) or upper extremity, which are supplied by the subclavian artery. Atherosclerosis is the most common cause of SSS, more common on the left side, may be due to a more acute origin of the left subclavian artery leads to increased

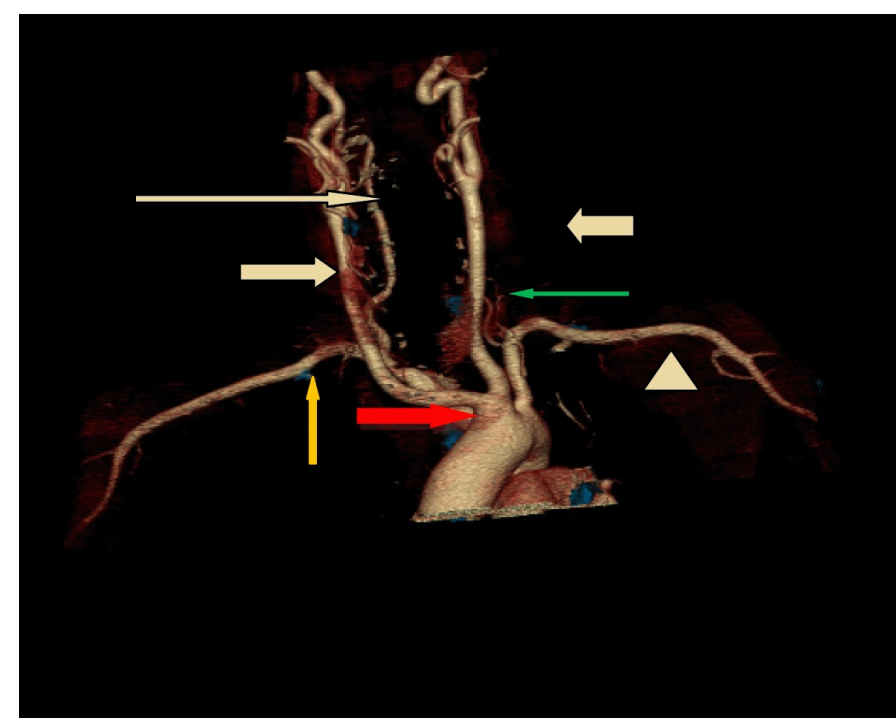

Figure 1: Three dimensionally reconstructed computed tomography angiography in anterior view showing common carotid trunk (red arrow) giving origin to left and right common carotid arteries (white arrows), aberrant right subclavian artery with stenosis proximally (yellow arrow), hypoplastic left vertebral artery (green arrow), normally originated right vertebral artery (long white arrow) and left subclavian artery (white triangle).

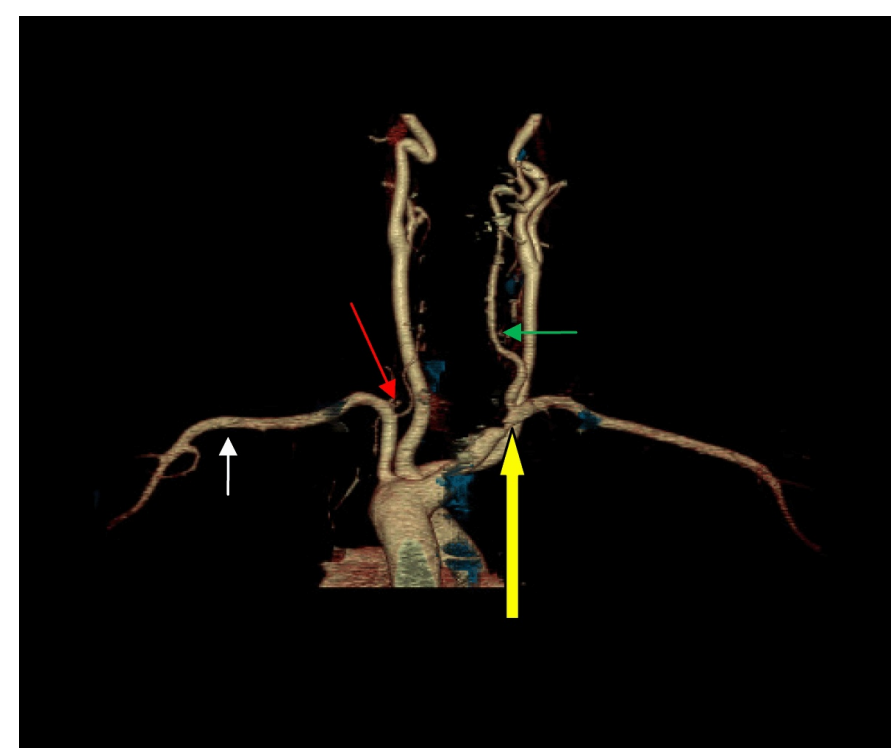

Figure 2: Three dimensionally reformatted computed tomography angiography in posterior view showing aberrant right subclavian artery with severe stenosis proximally (yellow arrow), hypoplastic left vertebral artery (red arrow), normally originated right vertebral artery (green arrow) and left subclavian artery (white arrow).

turbulence resulting in accelerated atherosclerosis [5]. Other conditions causing hemodynamically significant subclavian artery stenosis include Takayasu arteritis, compression of the subclavian artery in the thoracic outlet, following surgical repair of coarctation of the aorta and of tetralogy of Fallot with a Blalock-Taussig shunt and congenital abnormalities such as right aortic arch with isolation of the left subclavian artery and anomalies of the brachiocephalic arteries. Our patient had SSS due to stenosis in ARSCA. 


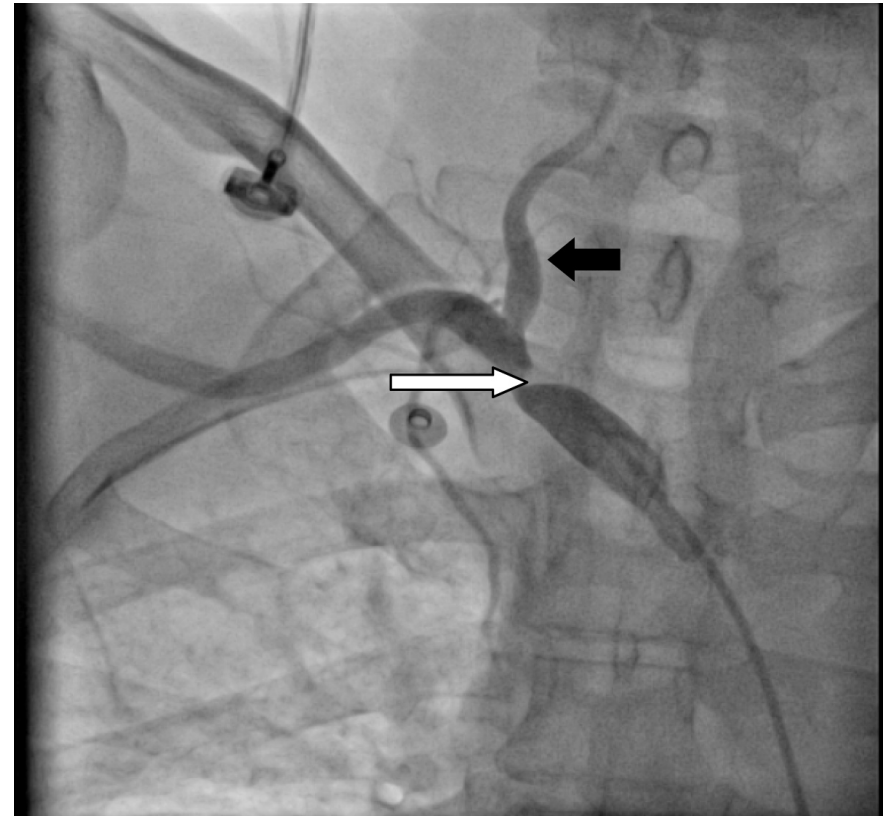

Figure 3: Selective angiogram of the aberrant right subclavian artery in right anterior oblique cranial view showing severe stenosis in its proximal part (white arrow) before the origin of right vertebral artery (black arrow).

The six pairs of aortic arches are a series of vessels that connect on each side the aortic sac with the corresponding dorsal aorta. At a later developmental stage, the aortic arches are both reduced in number and extensively transformed, and finally an asymmetric blood supply system is achieved (Figure 4A). It is understandable that many abnormalities can occur because of many changes involved the transformation of embryonic aortic arches into the adult arterial pattern. Most abnormalities result from the persistence of the parts of aortic arches that normally disappear or from disappearance of parts that normally persistent. Left aortic arch with an aberrant right subclavian artery, last great vessel on the aortic arch, is the most common congenital anomaly of the aortic arch occurring in about $0.5 \%$ of the general population [6]. Normally, proximal part of the right subclavian artery arises from the right fourth aortic arch and distal part from the right dorsal aorta present between fourth aortic arch and right seventh intersegmental artery and right seventh intersegmental artery (Figure 4A). The ARSCA develops as a result of the abnormal involution of the right fourth aortic arch, right dorsal aorta present between fourth aortic arch and seventh intersegmental artery and abnormal persistence of right dorsal aorta distal to the right seventh intersegmental artery (Figure 4B) [7]. It has been reported that the ARSCA typically travels in a retroesophageal course in $80 \%$ of patients like our patient, between the trachea and esophagus in 10-15\% of patients, and anterior to both structures in $5 \%$ of patients [8]. Most patients with an ARSCA remain asymptomatic. Only $10 \%$ of adult patients with ARSCA have symptoms [9].

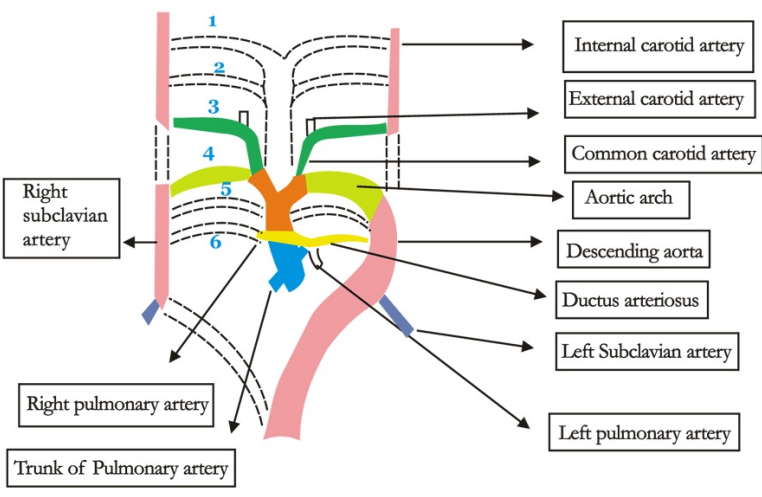

Truncus arteriosus $\square$ Aortic sac with left and right horns $\square 4^{\text {th }}$ arch artery

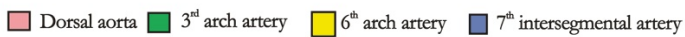

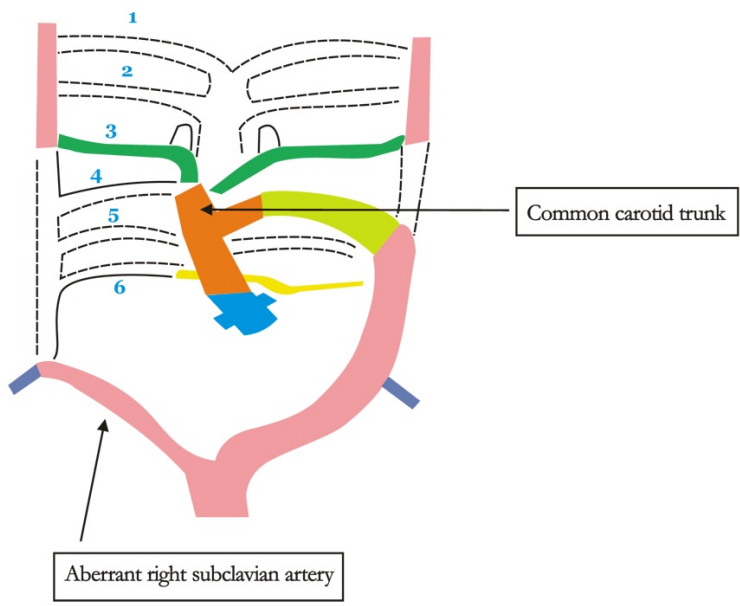

Figure 4: (A) Schematic drawing illustrating the arterial changes that result during transformation of the Truncus arteriosus, aortic sac, pharyngeal arch arteries, and dorsal aortas into the adult pattern. The vessels that are not colored will disappear during development, (B) Schematic drawing illustrating the development of aberrant right subclavian artery and common carotid trunk.

In 1993, Wells et al. reported that CCT is a left aortic arch variant and is present in about $13 \%$ of the general population [10]. During development, aortic sac, the most distal part of the truncus arteriosus, bifurcates into right and left horns, which forms the right brachiocephalic trunk and part of arch of aorta, respectively. Normally, third left aortic arch which forms the left common carotid artery and proximal part of the left internal carotid connected to the left horn of the aortic sac. The CCT arises when the proximal part of the third left aortic arch absorbed in to the right horn of the aortic sac. However, incomplete vascular ring caused by an ARSCA associated with CCT like our case is extremely rare and its incidence is about $0.16 \%$ [11].

Like our case one vertebral artery is hypoplastic in up to $10-15 \%$ of the healthy population and makes little 
contribution to basilar artery flow $[12,13]$. The left vertebral artery is dominant in approximately 50\%; the right in $25 \%$ and in the remaining $25 \%$ cases the two vertebral arteries are of similar calibre. Three prior studies concluded that individuals with hypoplastic vertebral arteries are at increased risk for posterior circulatory stroke more so if they had risk factors like hypertension, diabetes, smoking, etc. [14-16]. Our patient had hypoplastic left vertebral artery associated with stenosis in aberrant right subclavian artery proximal to the origin of right vertebral artery leading to relatively compromised blood flow to the brainstem and causing the vertebrobasilar symptoms with minimal exertion of the right upper extremity.

\section{CONCLUSION}

The combination of common carotid trunk, aberrant right subclavian artery and hypoplastic left vertebral artery in the same person was never reported earlier and this combination causes ischemia in large area of brain if the patient develops hemodynamically significant stenosis or occlusion in both the common carotid trunk and aberrant right subclavian artery proximal to the origin of right vertebral artery. All physicians should be aware of aortic arch variations prior to catheterization so that they can reduce fluoroscopic time and minimize contrast medium use.

\section{Author Contributions}

Boochi Babu Mannuva - Substantial contributions to conception and design, Acquisition of data, Drafting the article, Revising it critically for important intellectual content, Final approval of the version to be published Rajasekhar Durgaprasad - Substantial contributions to conception and design, Acquisition of data, Drafting the article, Revising it critically for important intellectual content, Final approval of the version to be published Vanajakshamma Velam - Substantial contributions to conception and design, Acquisition of data, Drafting the article, Revising it critically for important intellectual content, Final approval of the version to be published Shashanka Chunduri - Substantial contributions to conception and design, Acquisition of data, Drafting the article, Revising it critically for important intellectual content, Final approval of the version to be published Vijayalajshmi Devi B - Substantial contributions to conception and design, Acquisition of data, Drafting the article, Revising it critically for important intellectual content, Final approval of the version to be published

\section{Guarantor}

The corresponding author is the guarantor of submission.

\section{Conflict of Interest}

Authors declare no conflict of interest.

\section{Copyright}

(C) Boochi Babu Mannuva et al. 2013; This article is distributed under the terms of Creative Commons attribution 3.0 License which permits unrestricted use, distribution and reproduction in any means provided the original authors and original publisher are properly credited. (Please see www.ijcasereportsandimages.com /copyright-policy.php for more information.)

\section{REFERENCES}

1. Contorni L. The vertebro-vertebral collateral circulation in obliteration of the subclavian artery at its origin. Minerva Chir 1960;15:268-71.

2. Reivich M, Holling HE, Roberts B, Toole JF. Reversal of blood flow through the vertebral artery and its effect on cerebral circulation. $N$ Engl $J$ Med 1961;265:878-85.

3. Fisher CM. A new vascular syndrome: "The Subclavian Steal". N Engl J Med 1961;265:912-13.

4. Lord RS, Adar R, Stein RL. Contribution of the circle of Willis to the subclavian steal syndrome. Circulation 1969;40(6):871-8.

5. Kesteloot H, Vanhoute O. Reversed circulation through the vertebral artery. Acta Cardiol 1963;18:285-99.

6. Edwards JE. Malformations of the aortic arch system manifested as vascular rings. Lab Invest 1953;2(1):56-75.

7. Moor KL, Persaud TVN. The developing human. Clinically oriented embryology. 8 th ed. Philadelphia: W.B. Saunders; 2008:317-26.

8. Kadir S. Regional anatomy of the thoracic aorta. In: Kadir S,ed. Atlas of Normal and Variant Angiographic Anatomy. Philadelphia: WB Saunders; 1991:19-54.

9. Delap TG, Jones SE, Johnson DR. Aneurysm of an aberrant right subclavian artery presenting as dysphagia lusoria. Ann Otol Rhinol Laryngol 2000;109(2):231-4.

10. Wells TR, Landing BH, Shankle WR. Syndromal associations of common origin of the carotid arteries. Pediatr Pathol 1993;13(2):203-12.

11. Natsis KI, Tsitouridis IA, Didagelos MV, Fillipidis AA, Vlasis KG, Tsikaras PD. Anatomical variations in the branches of the human aortic arch in 633 angiographies: clinical significance and literature review. Surg Radiol Anat 2009;31(5):319-23.

12. Cloud GC, Markus HS. Diagnosis and management of vertebral artery stenosis. QJM 2003;96(1):27-54.

13. Jeng JS, Yip PK. Evaluation of vertebral artery hypoplasia and asymmetry by color-coded duplex ultrasonography. Ultrasound Med Biol 2004;30(5):605-9.

14. Park JH, Kim JM, Roh JK. Hypoplastic vertebral artery; frequency and associations with ischemic stroke territory. J Neurol Neurosurg Psychiatry 2007;78(9):954-8.

15. Chuang YM, Huang YC, Hu HH, Yang CY. Toward a further elucidation: role of vertebral artery hypoplasia in acute ischemic stroke. Eur Neurol 2006;55(4):193-7.

16. Perren F, Poglia D, Landis T, Sztajzel R. Vertebral artery hypoplasia: a predisposing factor for posterior circulation stroke? Neurology 2007;68(1):65-7. 
Access full text article on

other devices

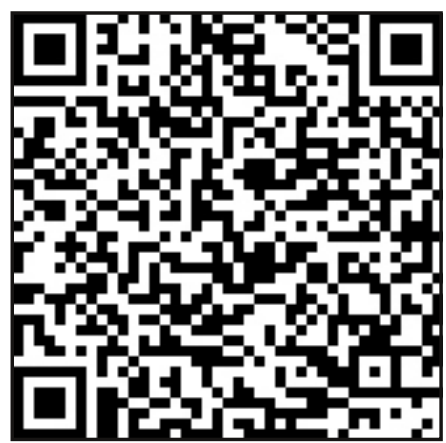

Access PDF of article on other devices

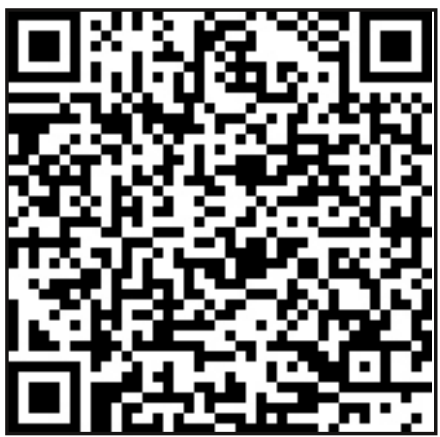

